\title{
Prognostic implications of stromal tumor-infiltrating lymphocytes and programmed death ligand 1 expression in HER2-positive invasive breast cancer
}

\section{Xinran Wang}

Hebei Medical University Fourth Affiliated Hospital and Hebei Provincial Tumor Hospital

Ningning Zhang

Hebei Medical University Fourth Affiliated Hospital and Hebei Provincial Tumor Hospital

Shi LI

Hebei Medical University Fourth Affiliated Hospital and Hebei Provincial Tumor Hospital

Yueping Liu ( $\square$ annama@163.com)

Hebei Medical University Fourth Affiliated Hospital and Hebei Provincial Tumor Hospital https://orcid.org/0000-0002-4582-114X

Research article

Keywords: breast cancer, TILs, PD-L1, HER2-positive

Posted Date: May 27th, 2020

DOl: https://doi.org/10.21203/rs.3.rs-29327/v1

License: (c) (i) This work is licensed under a Creative Commons Attribution 4.0 International License. Read Full License 


\section{Abstract}

Background: Tumor infiltrating lymphocytes (TILs) are known to be an important prognostic factor of specific breast cancer subtypes. However, to date, PD-L1(SP142)expression and tumor-infiltrating lymphocytes (TILs) has been only minimally reported in HER2-positive breast cancer cases.

Methods In this study, CD8,CD4 and PD-L1םSP142区 were performed on tissue microarrays (TMA) representing 156 pretreatment cases of HER2-positive invasive breast carcinoma, including 55 hormone receptor (HR)-negative BC patients diagnosed from January 2010 to September 2015.Clinical data were collected, all cases were with complete follow-up data (23 recurrence and 2 deaths). The concordance rate in the tumor immune microenvironment(TIME) classification based on the PD-L1 10SP142Пexpression status and CD8/CD4 + TILs count was analyzed.

Results PD-L1 expression was identified in 49 cases (31.4\%) in immune cells. We found there was no association was found between PD-L1 expression and DFS, but TIME classification was associated with DFS.

Conclusion CD4 + and CD8 + lymphocyte infiltration was related to DFS in HER2-positive breast cancer.

\section{Introduction}

Breast cancer is the major cause of cancer-related death among females worldwide ${ }^{[1]}$.Precise treatment based on molecular typing of breast cancer significantly improves the prognosis of patients.Approximately $20-25 \%$ of BCs have the human epidermal growth factor receptor 2 (HER2) gene amplification or protein overexpression. Anti-HER2 therapy, such as trastuzumab, represented a major breakthrough for patients with HER2-positive disease. However, Cancer cells and tumor-associated cells are phenotypically and functionally heterogeneous as a result of genetic and non-genetic sources,thus causing both primary and secondary resistances to anti-HER2 targets in about $50 \%$ of HER2-postive patients [2].

In recent years, immune therapy based on PD-1 (programmed death 1) or PD-L1 (programmed deathligand 1 ) identification by immunochemistry had become a hot topic ${ }^{[3]}$. Serving as an powerful immune checkpoint in tumor microenvironment, PD-1/ PD-L1 biomarkers had shown significant theropy efficacy in a variety of tumor types ${ }^{[4]}$.

Previously, most studies have implied that PD-L1 expression was associated with a worse prognosis in triple negative breast cancer, while tumor infiltrating lymphocytes (TILs) predicted favorable clinical outcomes, especially a abundant of CD8 + T cells both in triple negative and HER2-positive breast cancer $^{[5-6]}$. Recently, Vihervuori, et al. ${ }^{[7]}$ suggested that each $10 \%$ decrease in stromal TILs resulted in $20 \%$ increased risk of mortality. And, another research ${ }^{[8]}$ of $1318 \mathrm{BC}$ patients in European also suggested that tumor-infiltrating lymphocytes (TILs) density was remarkably related with PD-1 and PD-L1 
expression in immune cells. The importance of both PD-L1 expression and TILs prediction roles in TNBC of clinical outcome has been confirmed,but it reminds clinical research data in HER2-postive patients.

Recently, a study ${ }^{[9]}$ of lung adenocarcinoma patients which were divided into four subgroups based on the PD-L1 expression status and CD8 + TIL count in the tumors reported that the outcomes of patients with low PD-L1 expression levels and high CD8 + TIL counts were significantly better than those with high PD-L1 expression levels and low CD8 + TIL counts in the tumors, and this immunophenotyping were applied to breast cancer, shall we acquired the same result, we had analysed the result in our research.

The Food and Drug Administration (FDA) has recently granted an accelerated approval for atezolizumab, a monoclonal antibody drug targeting PD-L1, for the treatment of adults with PD-L1-positive TNBC, while SP142 is as the companion test for selecting TNBC patients, However, Limited data have been reported on the expression of PD-L1(SP142) in immune cells in a pure cohort of HER2-positive breast cancer, In the current study, we evaluated PD-L1(SP142) expression of immune cells and the relationship between TILs and PD-L1 or prognosis in HER2-positive cases. Moreover, an immune Classification of breast tumors into 4 groups on the basis of their PD-L1 status and CD4/CD 8 status has already been proposed in breast cancer for the first time.

\section{Materials And Methods}

\section{Patients and specimens}

A total of 156 confirmed cases of HER2-positive breast cancer paraffin specimens were collected from the Fourth Hospital of Hebei Medical University from Jan. 2010 to Sep. 2015.All cases were treated with standard adjuvant chemotherapy and HER2-blocking therapy following surgery. HER2 status was determined by HER2 immunohistochemistry (IHC) and/or HER2 fluorescence in situ hybridization (FISH).All cases with complete follow-up data with 23 recurrence and 2 deaths cases.

This study was conducted with the approval of the medical ethics review application form of Fourth Hospital of Hebei Medical University, which were used exclusively for research purposes in retrospective studies and in accordance with the Helsinki international declaration. Written informed consent for study of the excised tissue samples was obtained from each patient. All participants and/or legal guardians have obtained informed consent.

\section{Antibodies and Tissue microarrays}

Tissue microarray $(12 \star 8$ array) combined with immunohistochemistry was used to evaluate the expression of PD-L1(SP142) in tumor infiltrating lymphocytes (IC),3 cores size of $2 \mathrm{~mm}$ were collected as representative of primary resected tumor specimen.

Tissue microarray model was RP-20 (2.0 mm 8*12 points, Servicebio Company). IHC was performed for PD-L1 (SP142, Roche, Shanghai). Paraffin slices were baked in oven at $56^{\circ} \mathrm{C}$, dewaxed with xylene, hydrated with gradient ethanol, washed with phosphate buffer (PBS) and washed with distilled water. $3 \%$ 
of hydrogen peroxide solution blocked the endogenous peroxidase 10 min and EDTA antigen repair 15 min. A first antibody was added and incubated at $4{ }^{\circ} \mathrm{C}$ for the night. Rinse with PBS, add second anti drops, incubate 20-30 min at room temperature, rinse with PBS solution. Diaminobenzidine (DAB), hematoxylin re-staining, gradient ethanol dehydration, xylene transparency, neutral gum seals, observed under microscope.

\section{Pathologic assessment}

Tumor specimens from the 101 included patients were immunohistochemically evaluated to detect the expression of PD-L1 (SP142), CD4 and CD8. The stained tissue sections were independently scored by two pathologists who were blinded to the patients' clinical characteristics and outcomes.

PD-L1(SP142) positive expression was defined as any staining in more than $1 \%$ of immune cells(IC) and negative when less than $1 \%$ in the tumor area (Fig. 1). CD 4 and CD8 expression on lymphocytes was reported as the proportion of positive cells among all nucleated cells in the stromal compartments of each core, and scoring was recorded as negative $(<10 \%)$ or positive $(\geq 10 \%)$ (Fig. 1).

TILs are divided into intratumoral and stromal TILs. intratumoral TILs directly contact with tumor cells and there is no stromal tissue between cells while stromal TILs are distributed between tumor nests. Stromal TILs infiltration has been turned out more clinically significant. Therefore, this study mainly evaluates the infiltration of TILs in stromal tissue. The quantity of TILs in the sample was determined by the percentage of TILs covering the interstitial tissue area, and was defined as 1 , lymphocyte infiltration < $10 \% ; 2,10-40 \% ; 3, \varangle 40 \%$ (Fig. 2).

The TIME classification into four groups based on the PD-L1 and CD4/CD8 expression status has been proposed, as follows: type-I (PD-L1 positive expression with CD4/CD8 positive expression), type-II (PD-L1 negative expression with CD4/CD8 negative expression), type-III (PD-L1 positive expression with CD4/CD8 negative expression), and type-IV (PD-L1 negative expression with CD4/CD8 positive expression). And, the relationship between TIME and DFS was also analysed.

\section{Statistical analysis}

Data were analyzed by using SPSS 24.0 software. Qualitative variables were compared using Chi-square test and Fisher's exact test. Logistic regression was used for multivariate analysis. DFS was analysed firstly by Kaplan-Meier and log-rank test and later with Cox regression to adust for covariates. $P$-value were two sides and a $P<0.05$ was considered statistically significant. .

\section{Results}

\section{Patients Characteristics}

A total of 156 surgically resected FFPE primary HER2-positive breast carcinoma patients, including 55 hormone receptor (HR)-negative BC patients, were included in the study. The mean age at surgery was 
49 years (range, 28-73 years). The mean tumor size was $2.5 \mathrm{~cm}$ (range, $0.5-7.0 \mathrm{~cm}$ ) The majority of tumors $(63.5 \%)$ were grade 3 , while $50 \%$ of patients had lymph node metastasis and $45.5 \%$ of patients had lymphovascular invasion .Patients with a medium or high level of TILs accounted for $46.2 \%$. There was a median follow up of 57 months (range 36-104 months).(Table 1). 
Table 1

Clinical and pathological characteristics and immune reaction in 156 HER2positive breast cancers

\begin{tabular}{|c|c|c|c|}
\hline Characteristic & & Case $(n=156)$ & Percent (\%) \\
\hline \multirow[t]{2}{*}{ Age } & $\leq 50$ years & 69 & $44.2 \%$ \\
\hline & $>50$ years & 87 & $55.8 \%$ \\
\hline \multirow[t]{3}{*}{ Tumor size } & $\leq 2 \mathrm{~cm}$ & 67 & $42.9 \%$ \\
\hline & $\otimes 2, \leq 5 \mathrm{~cm}$ & 82 & $52.6 \%$ \\
\hline & $\nabla 5 \mathrm{~cm}$ & 7 & $4.5 \%$ \\
\hline \multirow[t]{2}{*}{ Histological garde } & प & 57 & $36.5 \%$ \\
\hline & ( & 99 & $63.5 \%$ \\
\hline \multirow[t]{2}{*}{ Lymphovascular invasion (LVI) } & Negative & 106 & $67.9 \%$ \\
\hline & Positive & 50 & $32.1 \%$ \\
\hline \multirow[t]{2}{*}{ Lymph node metastasis } & Negative & 85 & $54.5 \%$ \\
\hline & Positive & 71 & $45.5 \%$ \\
\hline \multirow[t]{2}{*}{ Ki67 } & $\leq 14 \%$ & 32 & $20.5 \%$ \\
\hline & $\bigotimes 14 \%$ & 124 & $79.5 \%$ \\
\hline \multirow[t]{2}{*}{ ER } & Negative & 57 & $36.5 \%$ \\
\hline & Positive & 99 & $63.5 \%$ \\
\hline \multirow[t]{2}{*}{ PR } & Negative & 74 & $47.4 \%$ \\
\hline & Positive & 82 & $52.6 \%$ \\
\hline \multirow[t]{2}{*}{ PD-L1 } & Negative & 107 & $68.6 \%$ \\
\hline & Positive & 49 & $31.4 \%$ \\
\hline \multirow[t]{3}{*}{ TILs } & $\bigotimes 10 \%$ & 84 & $53.8 \%$ \\
\hline & $\geq 10 \%, \varangle 40 \%$ & 57 & $36.6 \%$ \\
\hline & $\geq 40 \%$ & 15 & $9.6 \%$ \\
\hline \multirow[t]{2}{*}{ CD4 } & Negative & 93 & $59.6 \%$ \\
\hline & Positive & 63 & $40.4 \%$ \\
\hline \multirow[t]{2}{*}{ CD8 } & Negative & 104 & $66.7 \%$ \\
\hline & Positive & 52 & $33.3 \%$ \\
\hline
\end{tabular}




\section{Assessment of PD-L1 expression and immunereaction in 156 HER2-positive breast carcinomas}

Among all 156 cases, PD-L1 expression was identified in 49 cases(31.4\%). PD-L1 expression was positively associated with tumor size, Lymphovascular invasion ,TILs, CD4 + cells and CD8 + cells (Table 2); obtained using Logistic multivariate regression analysis the tumor size, the presence or absence of Lymphovascular invasion, the expression of TILs and CD8 were independent factors affecting PD-L1 expression (Table 3). 
Table 2

PD-L1 (SP142) expression and its association with clinicopathological characteristics in HER2-positive breast carcinoma

\begin{tabular}{|c|c|c|c|c|}
\hline \multicolumn{2}{|l|}{ Characteristic } & \multirow{2}{*}{$\begin{array}{l}\text { PD-L1-(N=107) } \\
50\end{array}$} & \multirow{2}{*}{$\begin{array}{l}\text { PD-L1+(N = 49) } \\
19\end{array}$} & \multirow{2}{*}{$\begin{array}{c}\boldsymbol{P} \\
0.353\end{array}$} \\
\hline Age & $\leq 50$ years & & & \\
\hline & $>50$ years & 57 & 30 & \\
\hline \multirow[t]{3}{*}{ Tumor size } & $\leq 2 \mathrm{~cm}$ & 53 & 14 & 0.028 \\
\hline & $\otimes 2, \leq 5 \mathrm{~cm}$ & 51 & 31 & \\
\hline & $\nabla 5 \mathrm{~cm}$ & 3 & 4 & \\
\hline \multirow[t]{2}{*}{ Histological grade } & $\square$ & 43 & 14 & 0.162 \\
\hline & $\square$ & 64 & 35 & \\
\hline \multirow[t]{2}{*}{ LVI } & Negative & 91 & 15 & $<0.001$ \\
\hline & Positive & 16 & 34 & \\
\hline \multirow[t]{2}{*}{ LNM } & Negative & 57 & 28 & 0.652 \\
\hline & Positive & 50 & 21 & \\
\hline \multirow[t]{2}{*}{ Ki67 } & $\leq 14 \%$ & 26 & 6 & 0.084 \\
\hline & $\otimes 14 \%$ & 81 & 43 & \\
\hline \multirow[t]{2}{*}{ ER } & Negative & 36 & 21 & 0.267 \\
\hline & Positive & 71 & 28 & \\
\hline \multirow[t]{2}{*}{ PR } & Negative & 50 & 24 & 0.794 \\
\hline & Positive & 57 & 25 & \\
\hline \multirow[t]{3}{*}{ TILs } & $\otimes 10 \%$ & 77 & 7 & $<0.001$ \\
\hline & $\geq 10 \%, \varangle 40 \%$ & 21 & 36 & \\
\hline & $\geq 40 \%$ & 9 & 6 & \\
\hline \multirow[t]{2}{*}{ CD4 } & Negative & 78 & 15 & $<0.001$ \\
\hline & Positive & 29 & 34 & \\
\hline \multirow[t]{2}{*}{ CD8 } & Negative & 89 & 14 & $<0.001$ \\
\hline & Positive & 18 & 35 & \\
\hline
\end{tabular}


Table 3

Multivariate logistic regression analysis of PD-L1 in HER2-positive breast carcinoma

\begin{tabular}{|lllllll|}
\hline Variable & B & SE & Wald & Sig. & $\operatorname{Exp}(\mathbf{B})$ & $95 \% \mathrm{Cl}$ \\
\hline Tumor size & 1.289 & 0.516 & 6.234 & 0.013 & 3.630 & $1.319-9.987$ \\
LVI & 1.749 & 0.632 & 7.665 & 0.006 & 5.751 & $1.667-19.840$ \\
TILs & 1.124 & 0.482 & 5.430 & 0.020 & 3.076 & $1.196-7.915$ \\
CD8 & 2.069 & 0.676 & 9.281 & 0.002 & 7.845 & $2.085-29.522$ \\
\hline \multicolumn{4}{|l}{ Note: LVI: Lymphovascular invasion } & & & \\
\hline
\end{tabular}

\section{The association of immune reaction and TIME classification with progression free survival (DFS)among HER2-positive breast carcinomas}

First, univariate analysis was performed to examine the associations between DFS and clinical/pathologic characteristics, including age, size, histological grades, Lymphovascular invasion, lymph node status, Ki67 index, ER and PR status, therapies and immune checkpoint expression parameters. The DFS was positively associated with tumor size, TILs, lymphovascular invasion, CD4 + cells, CD8 + cells, TIME-CD4 and TIME-CD8, but not other factors such as age, histological grade, lymph node metastasis, PD-L1 status ,ER/PR status, or Ki67 index (Table 4). Kaplan-Meier curves of DFS were also plotted with several significantly associated factors including with tumor size, TILs, lymphovascular invasion, CD 4 + cells, CD 8 + cells, TIME-CD 4 and TIME-CD8 as shown in Fig. 3. 
Table 4

Univariate analysis of factors associated with DFS in HER2-positive breast carcinoma

\begin{tabular}{|lll|}
\hline Variable & $\mathbf{X}$ & $\boldsymbol{P}$ \\
\hline Age & 3.011 & 0.083 \\
\hline Tumor size & 88.477 & $<0.001$ \\
\hline Histological garde & 0.598 & 0.439 \\
\hline LVI & 43.175 & $<0.001$ \\
\hline LNM & 6.304 & 0.012 \\
\hline Ki67 & 3.216 & 0.073 \\
\hline ER & 0.018 & 0.893 \\
\hline PR & 2.180 & 0.140 \\
\hline PD-L1 & 0.383 & 0.563 \\
\hline TILs & 12.213 & 0.002 \\
\hline CD4 & 17.754 & $<0.001$ \\
\hline CD8 & 14.780 & $<0.001$ \\
\hline TIME-CD4 & 32.454 & $<0.001$ \\
\hline TIME-CD8 & 48.339 & $<0.001$ \\
\hline Note: LVl: Lymphovascular invasion, LNM: Lymph node metastasis, & \\
\hline TIME-CD4: tumor immune microenvironment classification-CD4, \\
\hline TIME-CD8: tumor immune microenvironment classification-CD8 \\
\hline
\end{tabular}

Multivariate analysis with Cox regression model indicated that had lymphovascular invasion, TILs, TIMECD4 and TIME-CD8 were independent factor affecting DFS (Table 5). Moreover, in TIME-CD4 patients with PD-L1 negative expression and CD4 negative expression had a better prognosis while those with PD-L1 negative expression and CD4 positive expression had a worse prognosis; however in TIME-CD8 patients with PD-L1 positive expression and CD8 positive expression had a better prognosis while those with PDL1 positive expression and CD8 negative expression had a worse prognosis (Fig. 4). 
Table 5

Multivariate analysis of factors associated with DFS in HER2-positive breast carcinoma

\begin{tabular}{|lllllll|}
\hline Variable & B & SE & Wald & Sig. & Exp(B) & $95 \% \mathrm{Cl}$ \\
\hline Tumor size & 1.005 & 0.567 & 3.140 & 0.076 & 2.731 & $0.899-8.298$ \\
\hline LVI & 3.616 & 0.890 & 16.499 & $<0.001$ & 37.186 & $6.496-212.876$ \\
\hline LNM & 0.102 & 0.544 & 0.035 & 0.851 & 1.108 & $0.381-3.218$ \\
\hline TILs & 1.975 & 0.784 & 6.347 & 0.012 & 5.139 & $1.496-16.657$ \\
\hline CD4 & 1.265 & 0.384 & 3.035 & 0.081 & 3.545 & $0.854-14.720$ \\
CD8 & 2.014 & 1.217 & 2.737 & 0.098 & 0.133 & $0.012-1.451$ \\
\hline TIME-CD4 & 0.627 & 0.283 & 4.921 & 0.027 & 1.872 & $1.076-3.258$ \\
\hline TIME-CD8 & 0.853 & 0.310 & 7.563 & 0.006 & 2.346 & $1.278-4.309$ \\
\hline Note: LVI: Lymphovascular invasion, LNM: Lymph node metastasis, \\
\hline \multicolumn{7}{|l}{ TIME-CD4: tumor immune microenvironment classification-CD4, } \\
\hline \multicolumn{5}{|l}{ TIME-CD8: tumor immune microenvironment classification-CD8 } \\
\hline
\end{tabular}

\section{Discussion}

To our knowledge, the purpose of this cohort of HER2-positive breast cancer research was to evaluate the expression of PD-L1 in stromal I infiltrating immune cells and the infiltration level of tumor infiltrating lymphocytes (TILs) and the expression of other immunological biomarkers (CD4, CD8). Our study cohort included 156 patients who were HER2-positive. The expression rate of PD-L1 in 156 HER2-positive breast cancer mesenchymal immune cells in this study was $31.4 \%$ (49/156). In this study, it was found that the expression of PD-L1 in breast cancer with HER2-positive was significantly correlated with the tumor size, Lymphovascular invasion ,TILs infiltration level, CD4 + cells and CD8 + cells TILs mainly includes different types of $\mathrm{T}$ lymphocytes, B lymphocytes and NK cells, among which $\mathrm{T}$ lymphocytes that mainly play an anti-tumor role are divided into CD4 + T cells, CD8 + T cells and regulatory $T$ cells. The type and infiltration level of TILs are related to the prognosis of tumor patients ${ }^{[10-11]}$. In HER2-positive breast cancer, patients with high TILs infiltration levels often have a better prognosis ${ }^{[12]}$. Raphael et al. ${ }^{[13]}$ reported that in breast cancer with HER2-positive, the infiltration level of TILs was positively correlated with the histological grade, that is, the higher the histological grade was, the higher the infiltration level of TILs was. It suggested that TILs infiltration level was closely related to tumor differentiation. This study found that TILs infiltration level was positively correlated with the expression of PD-L1. Some studies have indicated that TILs infiltration level affects the responsiveness of breast cancer patients with HER2-positive to neoadjuvant chemotherapy and trastuzumab ${ }^{[14]}$. Therefore, the study of related factors affecting TILs in 
breast cancer and intervention may help improve the anti-tumor immune function of patients, improve the clinical tumor treatment effect and improve the prognosis of patients.

For breast cancer patients receiving standardized treatment, whether PD-L1 expression is a favorable or unfavorable prognostic factor is a subject of conflicting results in the literature. Most of the published studies have involved different subtypes of breast cancer, with the exception of several studies that specifically targeted TNBC ${ }^{[15,16]}$. Reported inconsistencies may be related to evaluation methods, numerical thresholds, antibody clones, or differences in cohort composition. In our cohort, patients' DFS was significantly correlated with ttumor size, TILs, lymphovascular invasion, CD4 + cells, CD8 + cells, TIME-CD4 and TIME-CD8,, while PD-L1 expression was not significantly correlated with patients' DFS. Since PD-L1 mediates checkpoint immune escape by suppressing the immune response of tumor cells, it has been speculated that the expression of PD-L1 may be associated with poor prognosis. However, studies of breast cancer and many tumors, including lung cancer and melanoma, have shown that PD-L1 expression is associated with better prognosis ${ }^{[17-19]}$.Therefore, the expression of PD-L1 in most cases may reflect a strong primary immune response rather than a successful immune escape. One possible explanation for these findings is that the expression of PD-L1 in immune cells in the tumors studied was an adaptive response to a cytotoxic immune attack on tumor neoantigens, rather than PD-L1 produced by tumor cells based on a gene activation pathway ${ }^{[20]}$. This will make these tumors more sensitive to driver pathway blocking therapy (such as HER2) or checkpoint immune pathway blocking therapy. The significance of different immune response patterns and intratumoral and periatumoral patterns for checkpoint blocking therapy must await data from immunotherapy trials. The biggest limitation of this study may lie in the use of tissue chips. TAMs may not be representative, increasing the possibility of false negative results.

In this study, we analyzed for the first time the relationship between immune typing and progression-free survival in HER2-positive patients. The results showed that the type of CD8+/PD-L1 + had the best prognosis, while the type of CD8-/PD-L1 + had the worst prognosis. The TILs infiltration score was positively correlated with prognosis. There are several limitations to our study. First, the number of patients participating in this study is small, reflecting the difficulty of obtaining the right number of matched samples for such studies. Second, for IHC analysis of PD-L1 expression and CD8 + TILs, we used a relatively standardized scoring system. However, different PD-L1 scoring schemes have been used to determine the treatment of each PD-L1 inhibitor available on the market. Although there is currently a standardized interstitial CD8 + TIL scoring system, there is no standardized CD8 + TIL scoring protocol for breast cancer..

In conclusion, our data indicate that the type of CD8+/PD-L1 + and TILs infiltration levels predict the prognosis of HER2-positive breast cancer treated with standard therapy. There are several shortcomings in this study: the number of cases was small, the tissue chip had some limitations, the heterogeneity of the tumor itself and the protein loss or insensitivity caused by the wax block stored for a long time. Some results have yet to be confirmed by big data. These findings, which are supported by other studies, offer hope for predicting immune checkpoint therapy.

Page $12 / 18$ 


\section{Declarations}

\section{Author contribution}

Yueping Liu,Xinran Wang,and Ningning Zhang collaborated in the study conception and design. Cases and blocks were selected from the Fourth Affiliated Hospital of Hebei Medical University by Xinran Wang, Ningning Zhang and Shi Li.Tissue microarrays were made by Danjing Yin.Data collection and analysis were performed by Xinran Wang and Ningning Zhang. The first draft of the manuscript was written by Xinran Wang and Ningning Zhang and all authors commented on previous versions of the manuscript. The whole experimental process was supervised and guided by Liu Yueping. All authors read and approved the final manuscript.

\section{Competing interests}

None declared.

\section{Provenance and peer review}

Not commissioned; externally peer reviewed

\section{References}

1. Duijf PHG, Nanayakkara D, Nones K,et al. Mechanisms of genomic instability in breast cancer. Trends Mol Med. 2019;25(7):595-611.

2. Goel S, Wang Q, Watt AC, et al. Overcoming Therapeutic Resistance in HER2-Positive Breast Cancers With CDK4/6 Inhibitors[J]. Cancer Cell. 2016;29(3):255-69.

3. Yasunaga M. Antibody Therapeutics and Immunoregulation in Cancer and Autoimmune Disease. Semin Cancer Biol. 2019..06.001.

4. Havel JJ, Chowell D, Chan TA. The evolving landscape of biomarkers for checkpoint inhibitor immunotherapy. Nat Rev Cancer. 2019;19(3):133-50.

5. Krishnamurti U, Wetherilt CS, Yang J, et al. Tumor-infiltrating lymphocytes are significantly associated with better overall survival and disease-free survival in triple-negative but not estrogen receptor-positive breast cancers $\mathbb{\bigotimes}[\mathrm{J}]$. Hum Pathol. 2017;64:7-12.

6. Hou Y, Nitta H, Wei L, et al. PD-L1 expression and CD8-positive T cells are associated with favorable survival in HER2-positive invasive breast cancer[J], 2018.;24(6):911-919.

7. Vihervuori $\mathrm{H}$, Autere TA, Repo $\mathrm{H}$,et al. Tumor infiltrating lymphocytes and CD8 + T cells predict survival of triple-negative breast cancer[J]. Journal of Cancer Research and Clinical Oncology,2019. ISSN (0171-5216). 
8. Noske A, Mobus V, Weber K, et al. Relevance of tumour-infiltrating lymphocytes, PD-1 and PD-L1 in patients with high-risk, nodal-metastasised breast cancer of the German Adjuvant Intergroup Nodepositive study. Eur J Cancer (Oxford, England: 1990). 2019;114:76-88.

9. Katsuhiko S, Riki O, Shinsuke S, et al. Prognostic value of Cox-2 and PD-L1 expression and its relationship with tumor-infiltrating lymphocytes in resected lung adenocarcinoma[J]. Cancer Management Research. 2017;9:741-50.

10. Bense RD, Sotiriou C, Piccart- Gebhart MJ, et al. Relevance of tumor-infiltrating immune cell composition and functionality for diseaseoutcome in breast cancer[J]. J Natl Cancer Inst. 2017;109(1):7-15.

11. Ali HR, Chlon L, Pharoah PD, et al. Patterns of immune infiltration in breast cancer and their clinical implications: a gene-expressionbased retrospective study[J]. PLoS Med. 2016;13(12):e1002194.

12. Barnes TA, Amir E. HYPE or HOPE: the prognostic value of infiltrating immune cells in cancer[J]. $\mathrm{Br} J$ Cancer. 2017;117(4):451-60.

13. Raphael J, Gong IY, Nofech-Mozes S, et al. Tumour infiltrating lymphocytes and stromal CD68 in early stage HER- 2 positive breast cancer[J]. J Clin Pathol. 2016;69(6):552-5.

14. Solinas $C$, Ceppi M, Lambertini M, et al. Tumor-infiltrating lymphocytes in patients with HER-2positive breast cancer treated with neoadjuvant chemotherapy plus trastuzumab,lapatinib or their combination: a meta- analysis of randomized controlled trials[J]. Cancer Treat Rev. 2017;57:8-15.

15. Baptista MZ, Sarian LO, Derchain SF, et al. Prognostic significance of PD-L1 and PD-L2 in breast cancer. Hum Pathol. 2016;47:78-84.

16. Cimino-Mathews A, Thompson E, Taube JM, et al. PD-L1 (B7-H1) expression and the immune tumor microenvironment in primary and metastatic breast carcinomas. Hum Pathol. 2016;47(1):52-63.

17. Dill EA, Gru AA, Atkins KA, et al. PD-L1 expression and intratumoral heterogeneity across breast cancer subtypes and stages: an assessment of 245 primary and 40 metastatic tumors. Am J Surg Pathol. 2017;41(3):334-342.

18. Velcheti V, Schalper KA, Carvajal DE, et al. Programmed death ligand-1 expression in non-small cell lung cancer. Lab Invest. 2014;94:107-116.

19. Taube JM, Anders RA, Young GD, et al. Colocalization of inflammatory response with $\mathrm{B} 7-\mathrm{H} 1$ expression in human melanocytic lesions supports an adaptive resistance mechanism of immune escape. Sci Transl Med. 2012;4(127):127ra37.

20. Mittendorf EA, Philips AV, Meric-Bernstam F, et al. PD-L1 expression in triple-negative breast cancer. Cancer Immunol Res. 2014;2:361-370.

\section{Figures}




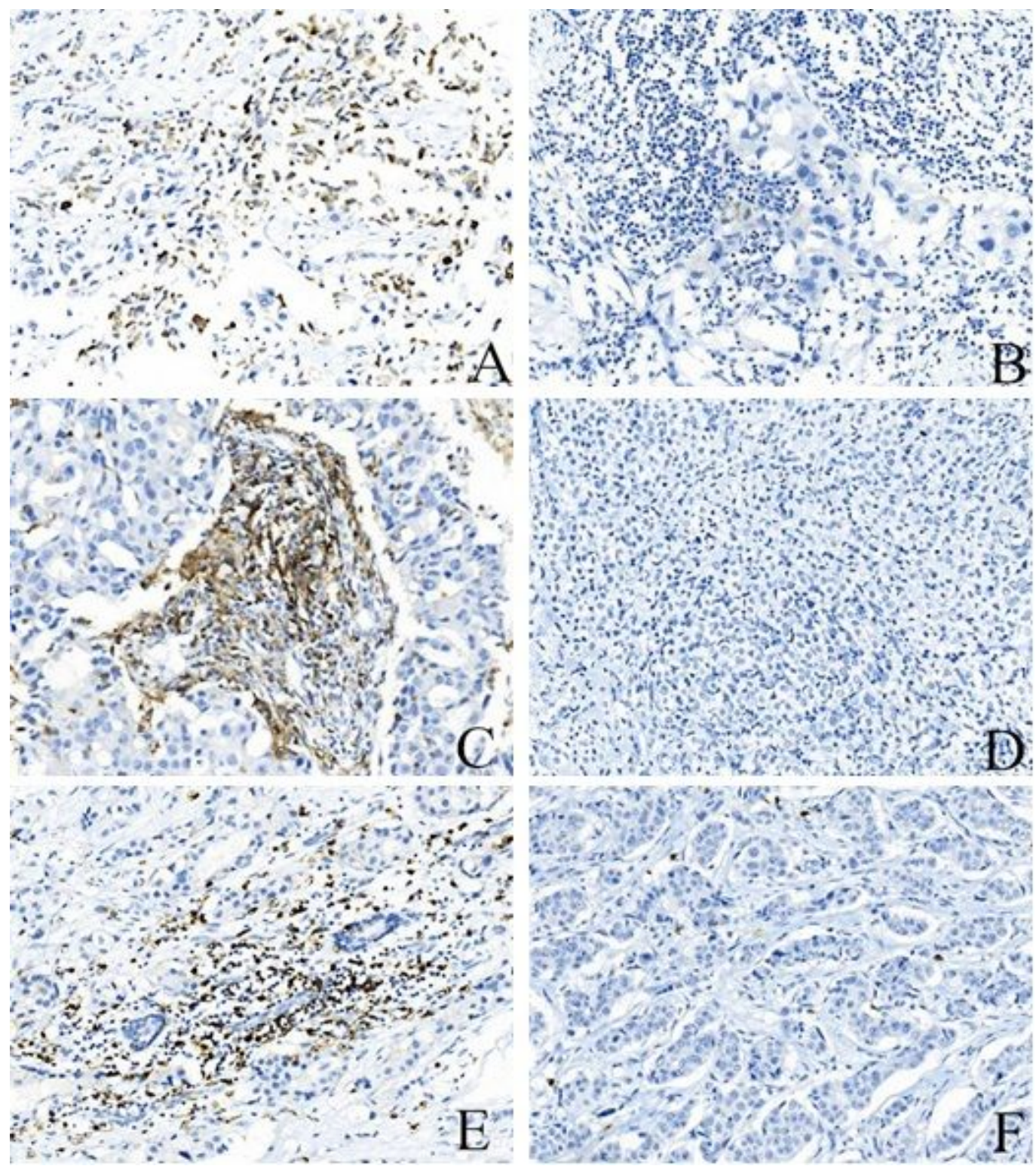

Figure 1

Expression of PD-L1 (SP142), CD 4 and CD8 antibodies in HER2-positive breast carcinoma $\ 10 \times \llbracket A$ : PD-L1 positive, B: PD-L1 negative, C: CD4 positive, D: CD4 negative, E: CD8 positive, F: CD8 negative 

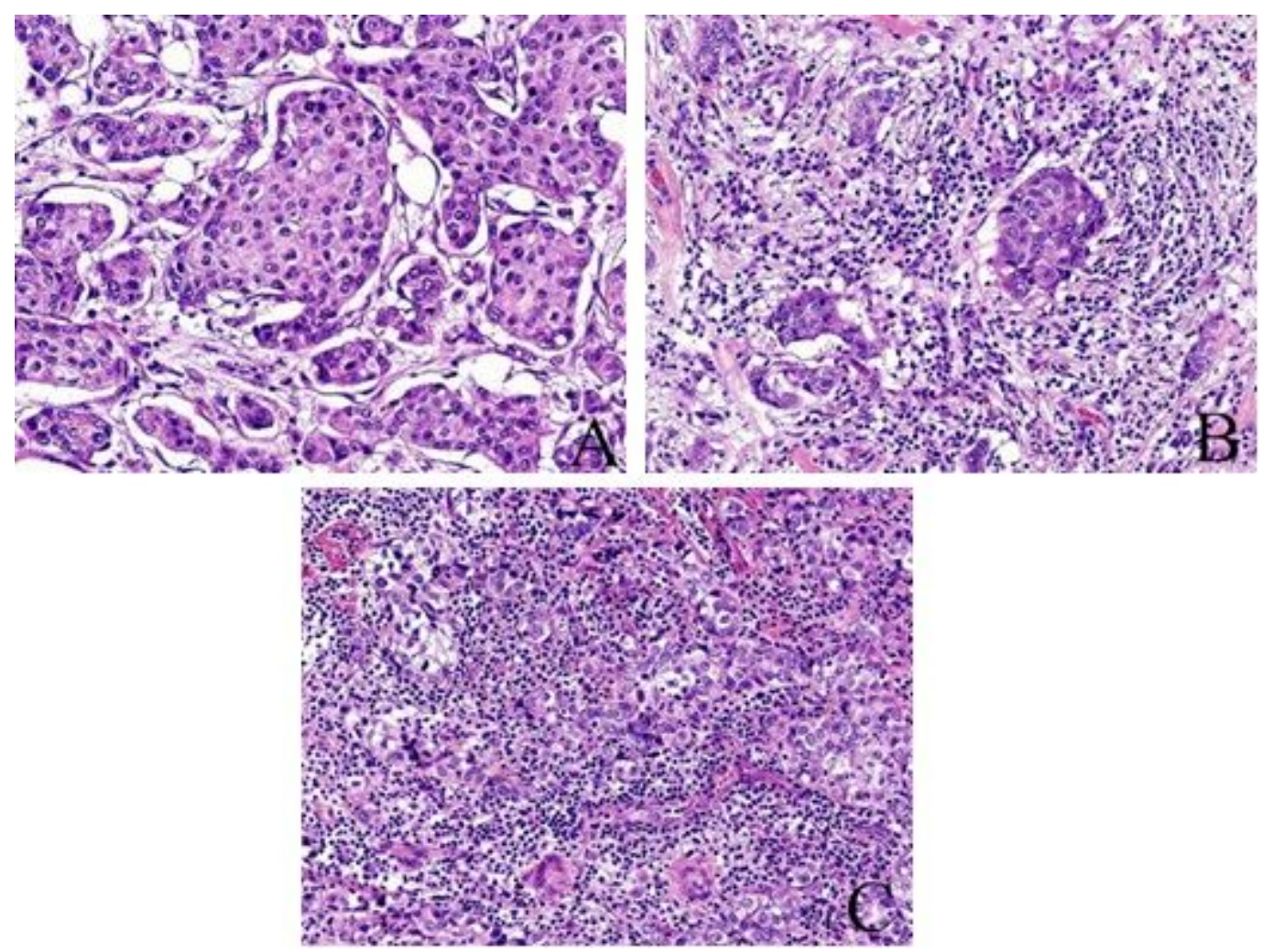

\section{Figure 2}

Representative microphotographs of sections from HER2-positive breast cancer samples showing the different tumour infiltrating lymphocyte (TILs) categories. A, TILs $<10 \% ; B, 10 \% \leq$ TILs $<40 \% ; C, T I L s \geq 40 \%$. 


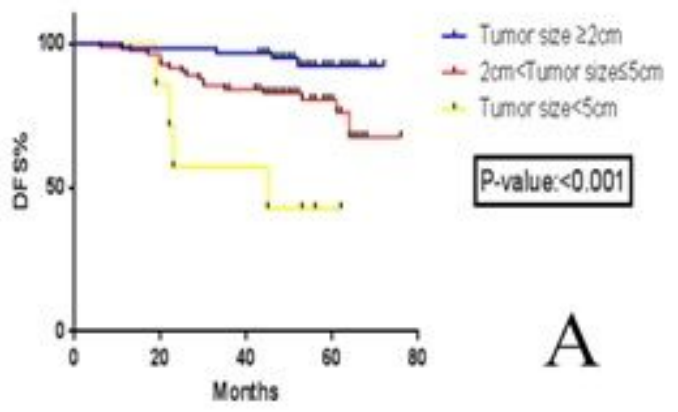

Disease Free Survival(DFS) accroding to LVI
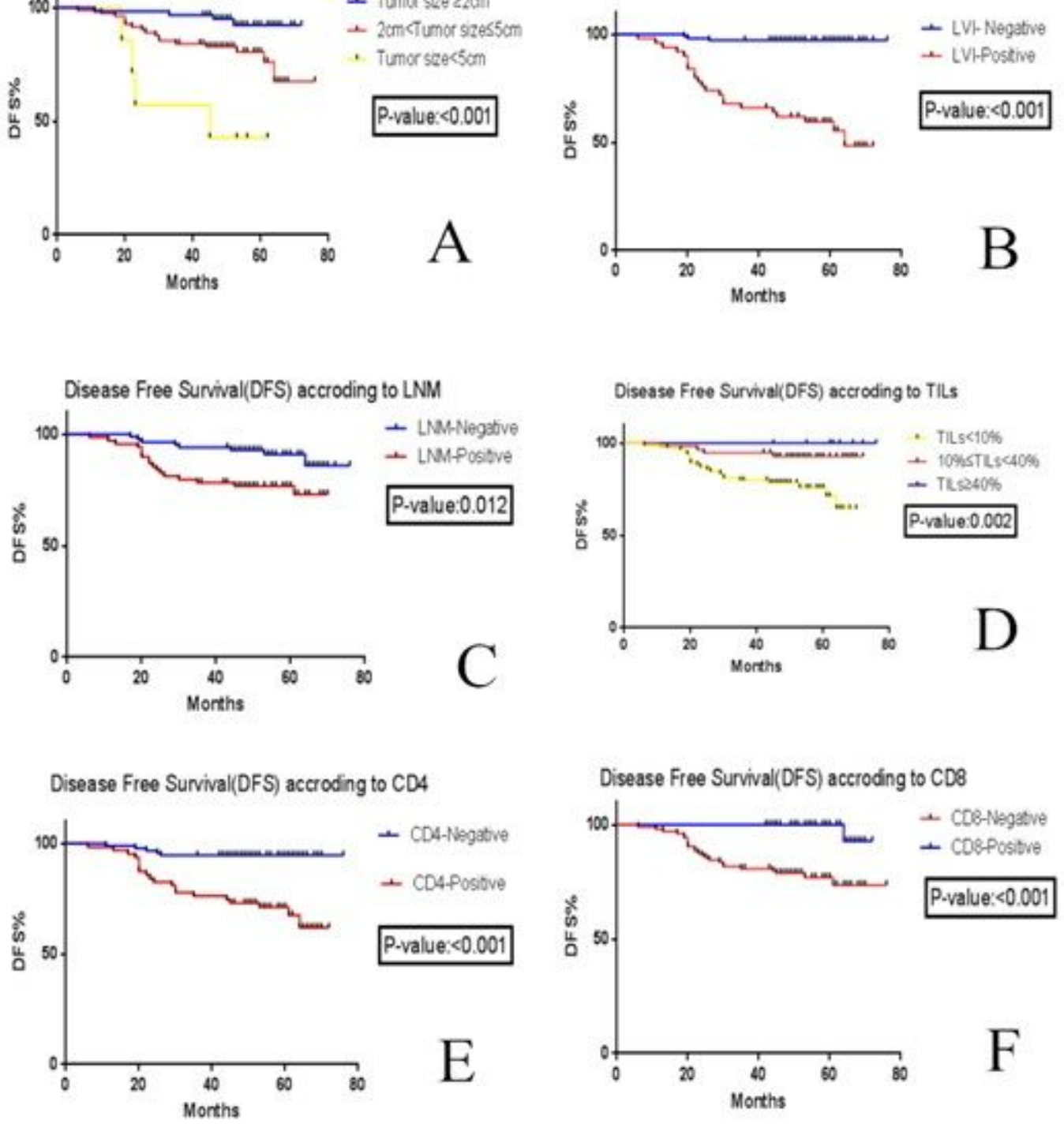

\section{Figure 3}

Effect of clinicopathological on DFS in HER2-positive breast carcinomas Kaplan-Meier survival analysis: A: Big tumor size is associated with poor prognosis; $\mathrm{B}$ : Lymphovascular invasion positive is associated with poor prognosis; C: Lymph node metastasis positive is associated with poor prognosis; D: Low TILs is associated with poor prognosis; E: CD4 positive expression is associated with poor prognosis; F: CD8 negative expression is associated with poor prognosis; 
Disease Free Survival(DFS) accroding to TIME -CD4

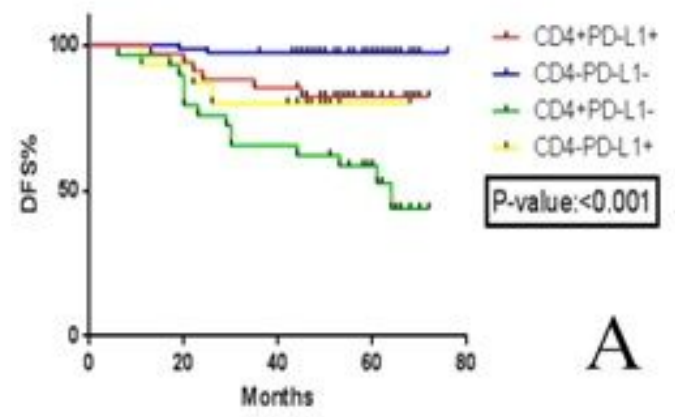

Disease Free Survival(DFS) accroding to TIME -CD8

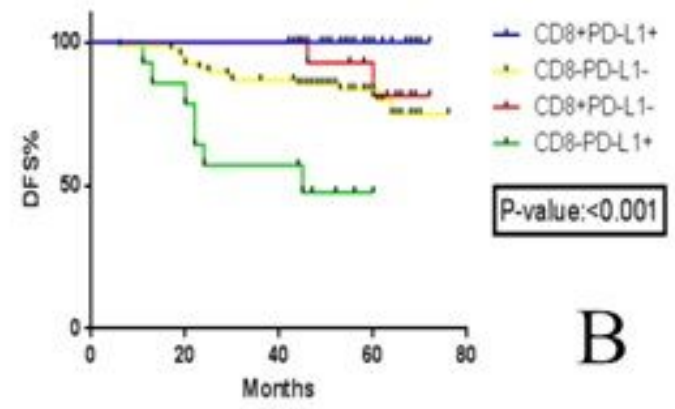

\section{Figure 4}

Effect of TIME on DFS in HER2-positive breast carcinomas Kaplan-Meier survival analysis: : CD4-/PD-L1with better prognosis; $\mathrm{B}$ : CD8+-/PD-L1+ is associated with better prognosis; 